\title{
Lateral resistance of ballasted tracks for various shapes of sleepers based on limit equilibrium methods
}

\author{
Takuma Ichikawa $^{\text {i) }}$, Kimitoshi Hayano ${ }^{\text {ii) }}$, Takahisa Nakamura ${ }^{\text {iii) }}$ and Yoshitsugu Momoya ${ }^{\text {iv) }}$ \\ i) Graduate student, Yokohama National University, 79-5, Tokiwadai, Hodogaya, Yokohama, Japan, 240-0065. \\ ii) Professor, Yokohama National University, 79-5, Tokiwadai, Hodogaya, Yokohama, Japan, 240-0065. \\ iii) Assistant senior researcher, Railway Technical Research Institute, 2-8-38, Hikari-cho, Kokubunji-shi, Tokyo, Japan, $185-8540$. \\ iv) Laboratory Head, Railway Technical Research Institute, 2-8-38, Hikari-cho, Kokubunji-shi, Tokyo, Japan, 185-8540.
}

\begin{abstract}
In this study, limit equilibrium methods were focused to evaluate lateral resistances of railway ballasted tracks. Lateral resistances under single-sleeper pull-out loadings as well as those under track-panel pull-out loadings were estimated for various shapes of sleepers. The lateral resistances were compared with those obtained from model tests. It was found that the limit equilibrium methods could well predict lateral resistances not only for a rectangular parallelepiped-shape sleeper but also for winged-shape sleepers having rectangular or trapezoid cross sections. It was also found that lateral resistances of various numbers of sleepers under track-panel pull-out loadings could be reasonably predicted by the methods.
\end{abstract}

Keywords: railway ballasted track, lateral stability, sleeper, limit equilibrium method

\section{INTRODUCTION}

It is quite important for railway ballasted tracks to have lateral resistances sufficient for the prevention of railway buckling. The lateral resistances are usually defined as resistance forces acting on sleepers when they are laterally pulled out in ballast beds.

To investigate the lateral resistance characteristics, laboratory model tests and/or full-scale field experiments have been conducted so far. For example, Koike et al. (2014) performed laboratory model tests in which $1 / 5$ scale modelled sleepers were pulled out. They revealed the effects of sleeper shapes and sleeper numbers on the lateral resistances.

They also proposed prediction methods to estimate lateral resistances of any numbers of sleepers under track-panel pull-out loadings. However, the methods were applicable only for lateral stabilities in the straight track sections, but could not be applied for those in the canted track sections. Moreover, the method could not consider earthquake effects on lateral resistances.

In views of above, limit equilibrium methods were focused in the present study to evaluate lateral resistances of ballasted tracks. Owing to the characteristics of the methods, it is expected that they will be easily implemented to evaluate lateral stabilities for canted tracks or tracks under seismic conditions in future studies.
L. M. Le Pen and W. Powrie (2011) proposed a limit equilibrium method and used it to evaluate lateral resistances of a single sleeper under single-sleeper pull-out loadings. Based on the method, in this study, lateral resistances of various shapes of sleepers not only under single-sleeper pull-out loadings but also those under track-panel pull-out loadings were estimated. The lateral resistances were compared with those obtained from model tests.

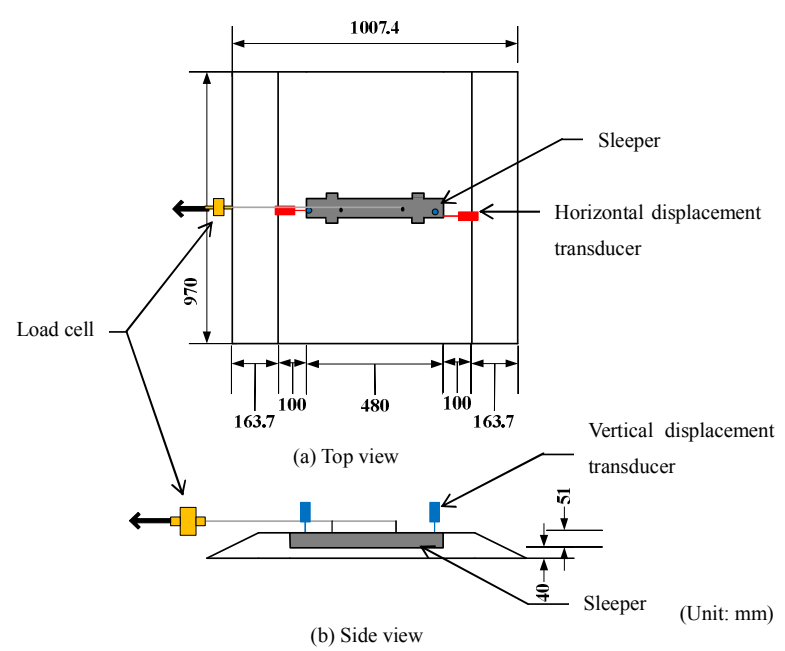

Fig. 1. Schematic image of single-sleeper pull-out loading tests (after Koike et al. 2014) 


\section{MODEL TESTS}

Fig. 1 shows an outline of the model tests conducted by authors (after Koike et al. 2014). The model tests were conducted on $1 / 5$ scale modeled sleepers. In addition to a conventional type sleeper called as $3 \mathrm{H}$ which is widely in the Shinkansen in Japan, a rectangular parallelepiped shape sleeper and winged-shape sleepers having rectangular or trapezoid cross sections were modeled. All the sleepers were made of mortar as shown in Fig. 2. The winged-shape sleepers had 20 or $40 \mathrm{~mm}$ long projection wings at the lateral sides of the sleepers. Ballasts in the model tests were crushed stones whose particles sizes were also $1 / 5$ of the actual ballasts. Ballast bed densities in all experiments were $1.60 \mathrm{~g} / \mathrm{cm}^{3}$.

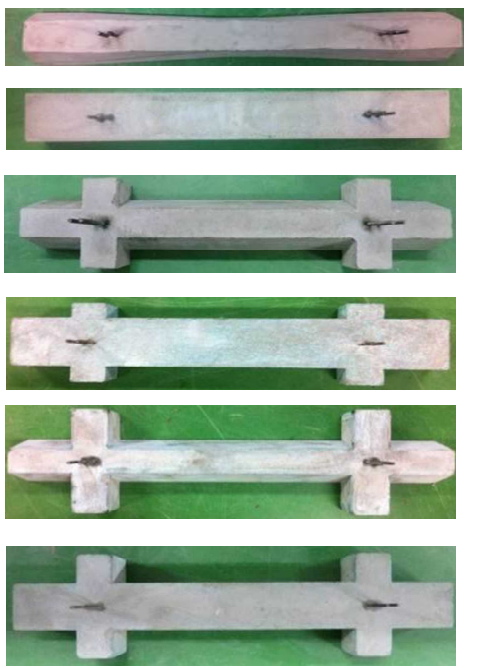

\section{H: 3H sleeper}

RP: Rectangular parallelepiped sleeper

W20 (tra): 20-mm-winged sleeper with a trapezoid section

W20 (rec): 20-mm-winged sleeper with a rectangular section

W40 (tra): 40-mm-winged sleeper with a trapezoid section

W40 (rec): 40-mm-winged sleeper with a trapezoid section

Fig. 2. Sleepers used for pull-out loading tests.

Single-sleeper pull-out loadings and track-panel pull-out loadings were performed on the above mentioned sleepers. The track-panel pull-out loadings displaced three or five or seven sleepers simultaneously with a rigid frame. The model test results showed the significant effects of sleeper shapes on the lateral resistances. Effects of sleeper numbers on the lateral resistances were also found from the track-panel pull-out loading tests. Some test results will be shown later to be compared with lateral resistances obtained by limit equilibrium methods.

\section{LIMIT EQUILIBRIUM METHOD}

Lateral resistance $R$ for a single sleeper was first evaluated based on the assumption that it was the sum of the contributions of three resistances as shown in Eq. (1). The resistances were the sleeper bottom resistance $R_{\text {bottom }}$, the sleeper side resistance $R_{\text {side }}$ and the sleeper end resistance $R_{\text {end }}$.

$$
R=R_{\text {bottom }}+R_{\text {side }}+R_{\text {end }}
$$

Each resistance could be evaluated as follows. For example, in case of a rectangular parallelepiped-shape sleeper, $R_{\text {bottom }}$ was evaluated by the following equation.

$$
R_{\text {bottom }}=\tan \delta_{\text {sleeper }} \times W_{\text {sleeper }}
$$

where, $W_{\text {sleeper }}$ was the sleeper weight and $\delta_{\text {sleeper }}$ was the angle of friction between the sleeper and ballasts. Here, $\delta_{\text {sleeper }}$ used in the evaluation was 42 degrees, which was directly obtained by pulling the sleeper laterally on the surface of ballast beds.

$R_{\text {side }}$ was evaluated by the following equation.

$$
R_{\text {side }}=2 \times \tan \delta_{\text {sleeper }} \times l \times \frac{1}{2} K_{0} \rho_{\mathrm{d}} h^{2}
$$

where, $l$ was the sleeper length, $K_{0}$ was the coefficient of earth pressure at rest, $\rho_{\mathrm{d}}$ was density of the ballast bed $\left(=1.60 \mathrm{~g} / \mathrm{cm}^{3}\right)$ and $h$ was the sleeper height. From the sleeper's geometry, $l$ and $h$ could be obtained, while $K_{0}$ could not be directly determined. Therefore, $K_{0}$ will be estimated based on the model test results as described later.

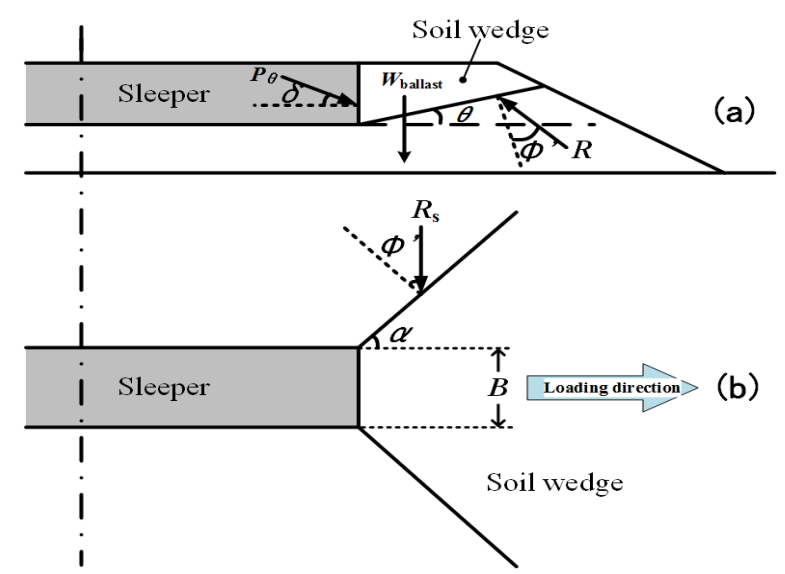

Fig. 3. Sliding soil wedge assumed for the evaluation of $R_{\text {end }}$

$R_{\text {end }}$ was evaluated based on a limit equilibrium method as shown in Fig. 3. Passive earth pressures acting on the sleeper end face with a sliding soil wedge were used for the evaluation. The angle between the slip lines of the sliding soil wedge and the sleeper's longitudinal direction $\alpha$ was set to be equal to the frictional angle of ballasts $\phi^{\prime}$ for simplifying the calculation. The frictional angle $\phi^{\prime}$ used in the calculation was 55 degrees, which was based on triaxial test results. Friction between the sleeper end face and ballasts $\delta_{\mathrm{w}}$ was required for the limit equilibrium method. It was changed in the range from 0 to $\delta_{\text {sleeper }}$ depending on the soil wedge shape. The details were described in L. M. Le Pen and W. Powrie (2011).

Eqs. (2) and (3) were also followed to estimate $R_{\text {bottom }}$ and $R_{\text {side }}$ of the other shape sleepers those were winged-shape sleepers and a $3 \mathrm{H}$ sleeper. To estimate $R_{\text {end }}$ of the winged-shape sleepers, the soil wedge whose slip 
lines starting from the wings' edges were assumed in the equilibrium method instead of those starting from the sleeper ends' edges as show in Fig.4. Moreover, the winged-shape sleepers denoted as W20 (tra) and W40 (tra) in Fig. 2 had a trapezoid cross section. Therefore, normal and shear stresses acting on the sleeper sides were calculated based on the side faces' inclination angles and they were considered in the estimation of $R_{\text {side }}$ and $R_{\text {bottom. }}$. Similarly, $R_{\text {side }}$ and $R_{\text {bottom }}$ of a $3 \mathrm{H}$ sleeper were obtained.

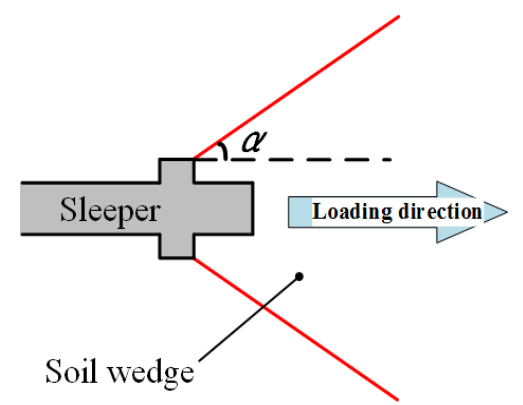

Fig. 4. Sliding soil wedge assumed in case of winged-shape sleepers

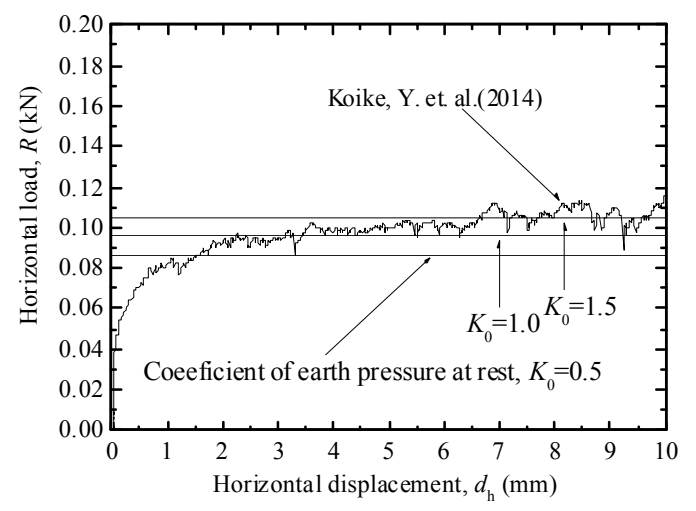

(a) Lateral resistance

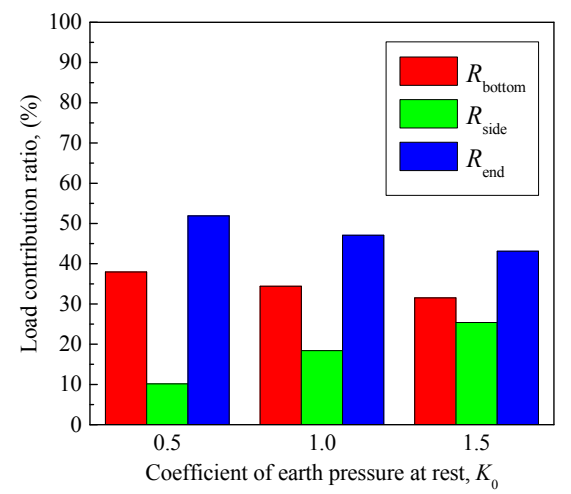

(b) Contributions of $R_{\text {bottom }}, R_{\text {side }}$ and $R_{\text {end }}$

Fig. 5. Effects of $K_{0}$ on the lateral resistance characteristics (case: Rectangular parallelepiped-shape sleeper)

\section{ANALYTICAL RESULTS COMPARED WITH MODEL TEST RESULTS}

4.1 Determination of $K_{0}$

As seen in Eq.(2), $K_{0}$ is required to estimate $R_{\text {side }}$. However, previous studies related to $K_{0}$ conditions of ballasts could not be found as far as authors' best knowledge. Therefore to find an appropriate $K_{0}$ value, lateral resistances were evaluated several times with changing $K_{0}$ values for the rectangular parallelepiped-shape sleeper denoted as RP (see Fig.2).

Fig. 5a shows the horizontal load and horizontal displacement relationship for the sleeper RP obtained from a single-sleeper pull-out loading test. The lateral resistances $R$ estimated by the limit equilibrium method in which $K_{0}$ are $0.5,1.0$ and 1.5 are also shown in the figure. It can be seen that the final lateral resistance measured in the model test is very close to that obtained by the limit equilibrium method where $K_{0}$ is 1.5 .

Fig. $5 \mathrm{~b}$ shows the contribution ratios of $R_{\text {bottom, }} R_{\text {side }}$ and $R_{\text {end }}$ to the lateral resistance $R$. It is seen the ratios depend on $K_{0}$. The contributions of $R_{\text {bottom, }} R_{\text {side }}$ and $R_{\text {end }}$ are $31.5 \%, 25.4 \%$ and $43.1 \%$ respectively when $K_{0}$ equals to 1.5 . The ratios were close to those measured in Sekine et al. 2010. The results shown in the figures suggest that model test results can be reasonably explained by the limit equilibrium method in which $K_{0}$ equals to 1.5 . Therefore in the study, the $K_{0}$ value is used in the estimation of lateral resistances hereafter.

4.2 Lateral resistances of various shapes of sleepers

Lateral resistances $R$ were obtained by the limit equilibrium method not only for the sleeper RP, but also for the sleepers 3H, W20 (rec), W20 (tra), W40 (rec) and W40 (tra) (see Fig.2). Those are plotted against the lateral resistances obtained from the single-sleeper pull-out loading tests in Figure 6. Here, lateral resistances $R$ in the model tests were defined as the horizontal resistance mobilized when the sleepers' horizontal displacement was $10 \mathrm{~mm}$. It is seen in the figure that the calculated lateral resistances are consistent well with the measured ones. The fact indicates that the limit equilibrium method can predict the lateral resistances for various shapes of sleepers if the sleeper shapes are appropriately handled in the method.

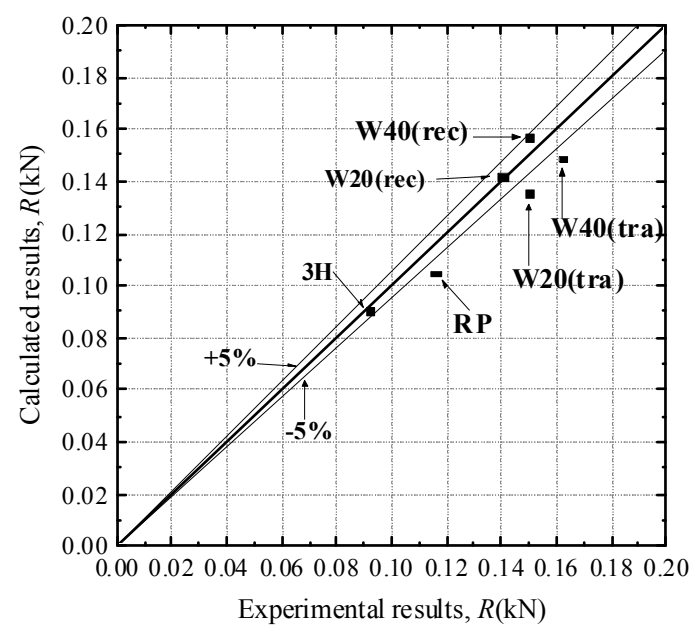

Fig. 6. Lateral resistances based on the limit equilibrium method plotted against those obtained from single-sleeper pull-out tests. 


\subsection{Lateral resistances of sleepers under track-panel} pull-out loadings

Koike et al.(2014) revealed the effects of sleeper numbers on the lateral resistances from the track-panel pull-out loading tests. Fig. 7 shows relationships between the horizontal resistance per one sleeper and the sleeper's displacement obtained from the track-panel pull-out loading tests. The sleeper type was $3 \mathrm{H}$. The track panel pull-out loading tests displaced three, five or seven sleepers simultaneously. The relationship obtained from the single-sleeper pull-out loading test is also shown in the figure. It is found that the lateral resistance per one sleeper was decreased with increase of sleeper numbers. The trend was also observed in case of the winged-shaped sleeper W20 (tra). It was considered that the phenomena were caused by piled group effects and boundary effects.

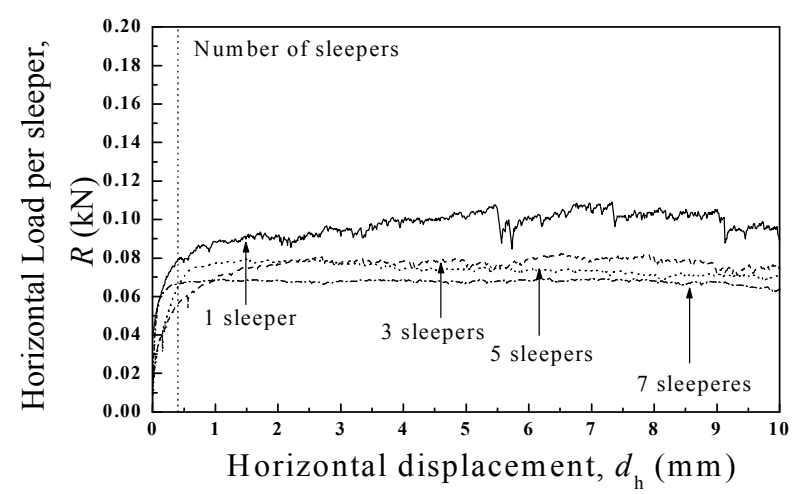

Fig. 7. Lateral resistance obtained from track panel pullout tests using different number of sleepers (case: $3 \mathrm{H}$ sleepers).

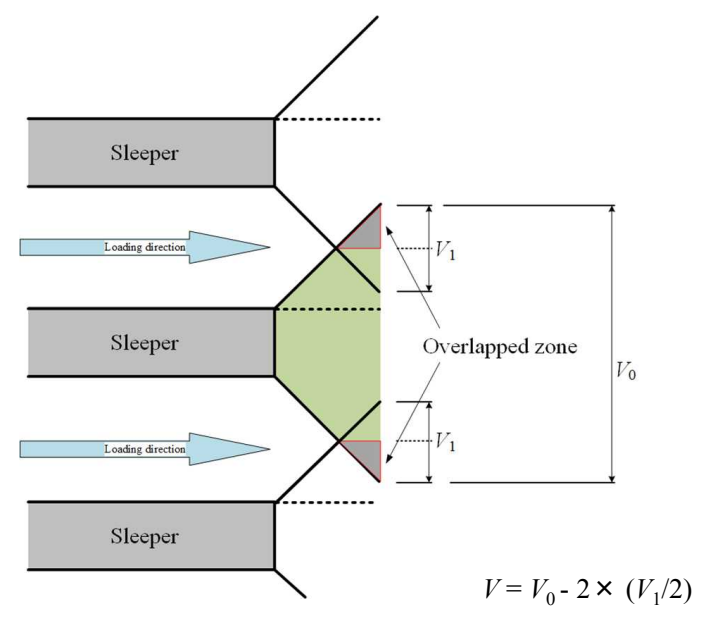

Fig. 8. Overlapping volumes of sliding soil wedges caused by the neighboring sleepers.

To consider the piled group effects on lateral resistances in the limit equilibrium method, the overlapping volumes of sliding soil wedges caused by the neighboring sleepers were calculated as shown in Fig. 8. Then, they were taken into account in the estimation of $R_{\text {end }}$. Fig. 9 shows the lateral resistances $R$ per one sleeper $3 \mathrm{H}$ or W20 (tra) calculated with the consideration of the piled group effects. They are plotted against numbers of sleepers subjected to pull-out loadings. The model test results are also shown in the figure. It is found that the calculated lateral resistances decrease with the increase of sleeper numbers, which tendency agrees well with the experiment results.

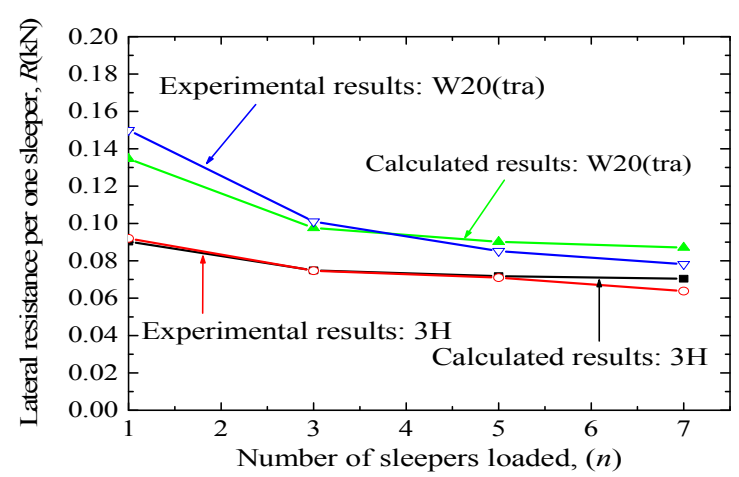

Fig. 9. Lateral resistance per one sleeper based on the limit equilibrium method plotted against the number of sleeper loaded in track panel pullout tests.

\section{CONCLUSIONS}

A limit equilibrium method was focused to evaluate the lateral resistance of ballasted tracks. The following findings are derived.

1. The limit equilibrium method could well predict the lateral resistance not only for a rectangular parallelepiped-shape sleeper but also for a $3 \mathrm{H}$ sleeper and winged-shape sleepers having rectangular or trapezoid sections.

2. The lateral resistance under track panel conditions could be reasonably predicted by the method.

\section{REFERENCES}

1) Ishikawa, T. et al. (1997), Cyclic deformation characteristics of railroad ballast in triaxial tests, Journal of Japanese Society for Civil Engineers, No. 575, 169-178 (in Japanese).

2) Koike, Y. et al. (2014): Numerical method for evaluating the lateral resistance of sleepers in ballasted tracks, Soils and Foundations, 54-3, 502-514.

3) Le Pen L. M. and Powrie W. (2011): Contribution of base, crib, and shoulder ballast to the lateral sliding resistance of railway track: a geotechnical perspective, Proceedings of the Institution of Mechanical Engineers, Part F, Journal of Rail and Rapid Transit, 225-2, 113-128.

4) Sekine, E. et al. (2010): Model tests on lateral resistances of ballasted tracks, Proceedings of 65th annual meetings of JSCE, No.4, 473-474 (in Japanese). 